\title{
Racial Disparities in End-of-Life Communication and Preferences among Chronic Kidney Disease Patients
}

\author{
Nwamaka D. Eneanya ${ }^{a} \quad$ Julia B. Wenger ${ }^{a}$ Katherine Waite $^{d}$ \\ Stanley Crittenden ${ }^{\mathrm{e}}$ Derya B. Hazar ${ }^{\mathrm{a}}$ Angelo Volandes ${ }^{\mathrm{b}}$ Jennifer S. Temel ${ }^{\mathrm{c}}$ \\ Ravi Thadhani $^{\mathrm{a}}$ Michael K. Paasche-Orlow ${ }^{\mathrm{d}}$ \\ a Division of Nephrology, ${ }^{b}$ Division of General Medicine, and ${ }^{c}$ Division of Hematology and Oncology, \\ Department of Internal Medicine, Massachusetts General Hospital, Harvard Medical School, ' Section of General \\ Internal Medicine and 'Division of Nephrology, Boston Medical Center, Boston University School of Medicine, \\ Boston, Mass., USA
}

\section{Key Words}

Racial disparities · End-of-life · Chronic kidney disease

\begin{abstract}
Background: Previous studies on end-of-life (EOL) care among patients with chronic kidney disease (CKD) have been largely limited to White hemodialysis patients. In this study, we sought to explore racial variability in EOL communication, care preferences and advance care planning (ACP) among patients with advanced CKD prior to decisions regarding the initiation of dialysis. Methods: We performed a cross-sectional study between 2013 and 2015 of Black and White patients with stage IV or V CKD (per the Modified Diet in Renal Disease estimation of GFR $<30 \mathrm{ml} / \mathrm{min} / 1.73 \mathrm{~m}^{2}$ ) from 2 academic centers in Boston. We assessed experiences with EOL communication, ACP, EOL care preferences, hospice knowledge, spiritual/religious and cultural beliefs, and distrust of providers. Results: Among 152 participants, 41\% were Black. Black patients were younger, had less education, and lower income than White patients (all $p<0.01$ ). Black patients also had less knowledge of hospice compared to White patients ( 17 vs. $61 \%, p<0.01$ ). A small fraction of pa-
\end{abstract}

tients (8\%) reported having EOL discussions with their nephrologists and the majority had no advance directives. In multivariable analyses, Blacks were more likely to have not communicated EOL preferences (adjusted OR $2.70,95 \% \mathrm{Cl}$ 1.08-6.76) and more likely to prefer life-extending treatments (adjusted OR 3.06, 95\% Cl 1.23-7.60) versus Whites. Conclusions: As Black and White patients with advanced CKD differ in areas of EOL communication, preferences, and hospice knowledge, future efforts should aim to improve patient understanding and promote informed decisionmaking.

(c) 2016 S. Karger AG, Basel

\section{Introduction}

Although survival rates for patients with chronic kidney disease (CKD) and end-stage renal disease (ESRD) initiating hemodialysis (HD) have slightly improved, only $54 \%$ of people who start HD are alive within 3 years [1]. Furthermore, patients with ESRD typically experience intensive patterns of healthcare utilization at the end-of-life (EOL) as opposed to comfort care [2]. For in-

\section{KARGER}

E-Mail karger@karger.com

www.karger.com/ajn
(C) 2016 S. Karger AG, Basel

0250-8095/16/0441-0046\$39.50/0
Nwamaka D. Eneanya, MD, MPH

Division of Nephrology, Department of Internal Medicine

165 Cambridge Street, Suite 302

Boston, MA 02114 (USA)

E-Mail Aeneanya@ partners.org 
stance, many who choose dialysis also receive other lifesustaining treatments such as cardiopulmonary resuscitation and mechanical ventilation despite a poor likelihood of survival [3-5]. As communication about care desired at the EOL is one of the best ways for people to preserve their wishes, early goals of care discussions are especially salient in this population [6-9].

Previous literature on EOL conversations and preferences for care among patients with CKD has largely been limited to patients on maintenance HD [6, 10-12]. Despite national guidelines and initiatives to improve EOL care for nephrology patients, recent studies have demonstrated that patients infrequently engage in prognostic and EOL discussions with their nephrologists $[6,7,13]$. Furthermore, studies of advance care planning (ACP), defined as the process where individuals plan ahead for care desired if they were to become incapacitated, and patients with kidney disease have been conducted almost exclusively among White patients $[6,14-16]$. Several disciplines of healthcare have demonstrated how minority racial-ethnic groups typically experience less access to palliative care services, receive more life-sustaining therapies and are less likely to use hospice during the final months of life compared to other racial-ethnic groups [17-21]. While poor education, spirituality, and trust of the healthcare system are likely to contribute to disparities in patterns of care at the EOL, such relationships have not been evaluated in CKD patients $[21,22]$. In this study we sought to explore whether racial variability exists with regards to EOL communication, care preferences, and ACP among patients with advanced CKD prior to initiation of dialysis.

\section{Methods}

\section{Setting and Study Participants}

We performed a cross-sectional study between August 2013 and February 2015 among patients with stage IV or V CKD (as defined by the Modified Diet in Renal Diseases [23] estimation of GFR $<30 \mathrm{ml} / \mathrm{min} / 1.73 \mathrm{~m}^{2}$ ). Patients were recruited from outpatient nephrology clinics associated with 2 academic centers in Boston, Massachusetts. Eligibility included age 45 or older, Englishspeaking and self-report of Black or White race. Patients were excluded if they had a known history of dementia or were found to have severe cognitive deficit (as determined by 8 or more errors on the Short Portable Mental Status Questionnaire) [24]. Patients were also excluded if they were listed for kidney transplantation as the illness trajectory and prognoses for such patients differs significantly from those without this treatment option. The Institutional Review Boards at Partner's Healthcare and Boston Medical Center approved this study.

Racial Disparities in EOL Care among CKD Patients

\section{Data Collection}

All study personnel underwent training in conducting structured patient interviews using study questionnaires. All interviews were performed in a quiet and private room in the outpatient clinic at a scheduled routine visit. Patient demographic information including age, gender, race, ethnicity, formal level of education, annual income, and health insurance status were ascertained through study questionnaires. We reviewed electronic medical records (EMRs) for comorbid conditions and the Charlson Comorbidity Index (CCI) score was calculated [25].

\section{Outcomes}

The primary outcome was prior EOL communication with any providers (yes vs. no). Additional outcomes included, EOL communication with nephrologists (yes vs. no), EOL communication with family members or friends (yes vs. no), ACP (possession of healthcare proxy (HCP) and Do-Not-Rescusitate (DNR) forms or living wills; yes vs. no) and EOL preferences. As previously done in research investigating EOL care in seriously ill patients [26], we ascertained EOL preference for resuscitation (yes vs. no) as well as life-extending care (comfort vs. life-extending) and site of death (hospital vs. home) at the EOL. Using the outpatient EMR, we verified whether DNR orders, living wills, and HCP forms had been completed for all patients. For patients who had evidence of these forms in the EMR, they were given credit for having such documentation even if this had not been reported. For any missing information in the EMR, we deferred to the patient's response as confirmation.

We collected secondary outcomes including spiritual/religious beliefs, cultural beliefs, and distrust of healthcare providers and their influence on EOL preferences using single-item measures (yes vs. no) during patient interviews. In addition, hospice knowledge was determined by content analysis of an open-ended, follow-up item for participants who had heard of the term 'hospice' ('since you have heard of hospice, what does this mean to you in your own words?'). Three members of the study staff subsequently and independently categorized responses into 'poor knowledge', 'partial knowledge', or 'good knowledge' [27]. The third study staff member was also used to adjudicate any disagreements in categorization.

\section{Statistical Analyses}

Descriptive participant characteristics are presented using proportions for categorical means and standard deviations (SDs) for continuous variables. We tabulated the proportions of participants who had a history of EOL discussion with their healthcare providers/families, preference for resuscitation, EOL care and site of care, and possession of HCP form, DNR order or living will. We also ascertained whether culture, religion/spirituality and distrust of healthcare providers had an impact on preferences. Hospice knowledge response categories were dichotomized into poor or partial knowledge versus good knowledge.

We used univariate logistic regression analysis to identify statistically significant racial differences in EOL preferences and communication. Models were examined for each of 5 outcomes, where statistically significant racial differences existed in univariate analysis including: (1) no communication of EOL preferences with family; (2) no possession of an HCP form; (3) no possession of DNR order or living will; (4) preference for EOL life-extending care; and (5) preference for site of death. Collinearity for demo- 
graphic variables, hospice knowledge, and study site with race were tested using the variance inflation factor. Multivariable logistic regression analyses were then performed to identify significant predictors of each of the 5 outcomes. As the distribution of elderly patients differed among racial groups, we stratified the final analyses by age ( $<65$ vs. $\geq 65$ years). In addition, we stratified the final analysis of hospice knowledge by the level of education. All analyses were performed using SAS version 9.2 (SAS Institute Inc., Cary, N.C., USA). Statistical significance was determined by $\mathrm{p}$ values $<0.05$.

\section{Results}

\section{Patient Characteristics}

A total of 268 patients were approached for study enrollment. Fifty-four patients were ineligible, 61 patients declined participation, and one patient stopped the study early (fig. 1). Among 57\% of patients who enrolled and completed the study, $41 \%$ were Black. Black patients were younger compared to White patients with a mean age \pm SD of $66 \pm 11$ vs. $70 \pm 10(p=0.01$; table 1$)$. A higher proportion of Black patients had an annual income $<\$ 30,000$ and a lower level of education attainment (both $\mathrm{p}<0.01$ ). There was no significant difference in mean CCI score between the 2 racial groups.

\section{Hospice Knowledge}

All White patients $(n=89)$ and $75 \%$ of Black patients $(\mathrm{n}=47)$ reported that they had heard of 'hospice' and responded to the query about the meaning of hospice. Examples of these patients' verbatim descriptions of the meaning of hospice are listed in table 2 . We determined $17 \%(8 / 47)$ of Black patients' and $61 \%(54 / 89)$ of White patients' responses as exhibiting good knowledge of hospice $(\mathrm{p}<0.01)$. In addition, when stratified by level of education, we observed that among those who graduated from a high school $(\mathrm{n}=128)$, racial differences persisted $(p<0.01)$, but these differences were not significant among those who had not graduated from a high school $(\mathrm{n}=24, \mathrm{p}=0.42)$.

\section{Previous EOL Communication}

Overall, 77\% percent of patients reported never having a prior EOL discussion with any healthcare provider (table 1). Specifically, $92 \%$ of patients reported never having such conversations with a nephrologist. Significantly more Black patients reported not having discussions about EOL preferences with their family members or friends compared to White patients ( 54 vs. $27 \%, \mathrm{p}=0.01$; table 1). After adjusting for age, education, income, study

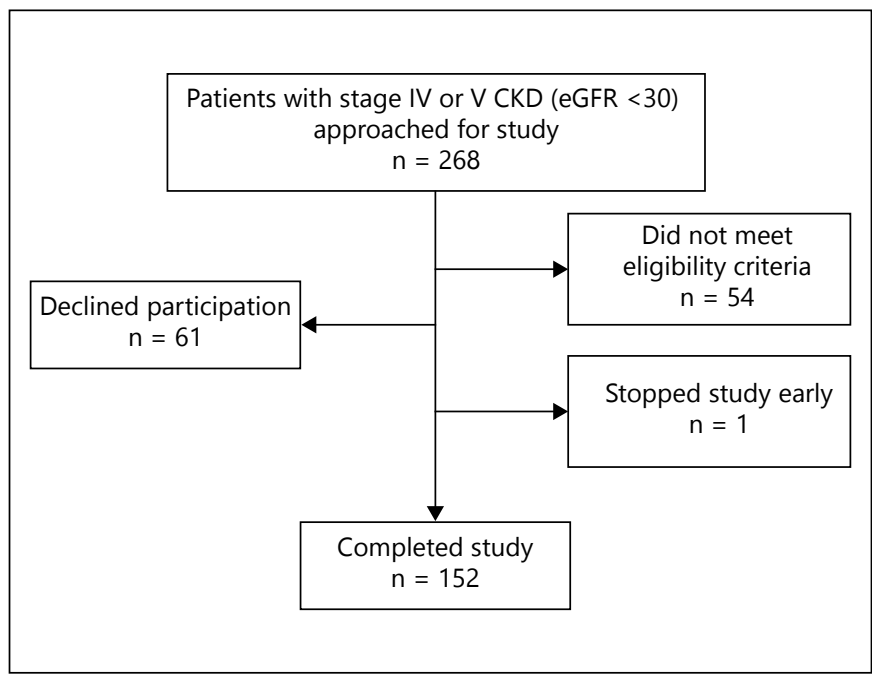

Fig. 1. Study enrollment.

site, CCI score and hospice knowledge, Black patients were still more likely to not communicate EOL preferences with family members and friends (adjusted OR 2.70, 95\% CI 1.08-6.76; table 3) compared to White patients.

\section{Advance Care Planning}

Two patients reported that they did not have a living will and 14 patients reported having no HCP despite record of these documents in the EMR. There were no differences in patient characteristics between those with and without EMR evidence of ACP documentation. A higher proportion of Black patients reported that they had not completed DNR orders or living wills and had no evidence of such documentation in the EMR compared to White patients ( 78 vs. $62 \%, \mathrm{p}=0.04$; table 1 ). More Black patients also had not completed HCP forms and had no evidence of such documentation in the EMR compared to White patients ( $50 \mathrm{vs.} 30 \%, \mathrm{p}=0.01$ ). In adjusted analyses, there were no significant racial differences.

\section{EOL Care Preferences}

A total of $89 \%$ of patients preferred to be resuscitated and there was no difference in this preference between Blacks and Whites. At the same time, a higher proportion of Black patients preferred to extend life as opposed to focusing on comfort care compared to White patients (56 vs. $25 \%, \mathrm{p}<0.01$; table 1). Similarly, Blacks were more likely to prefer spending their final moments of life in a hospital vs. Whites (41 vs. $19 \%, \mathrm{p}<0.01$ ). In adjusted
48

Am J Nephrol 2016;44:46-53 DOI: $10.1159 / 000447097$
Eneanya et al. 
Table 1. Patient characteristics

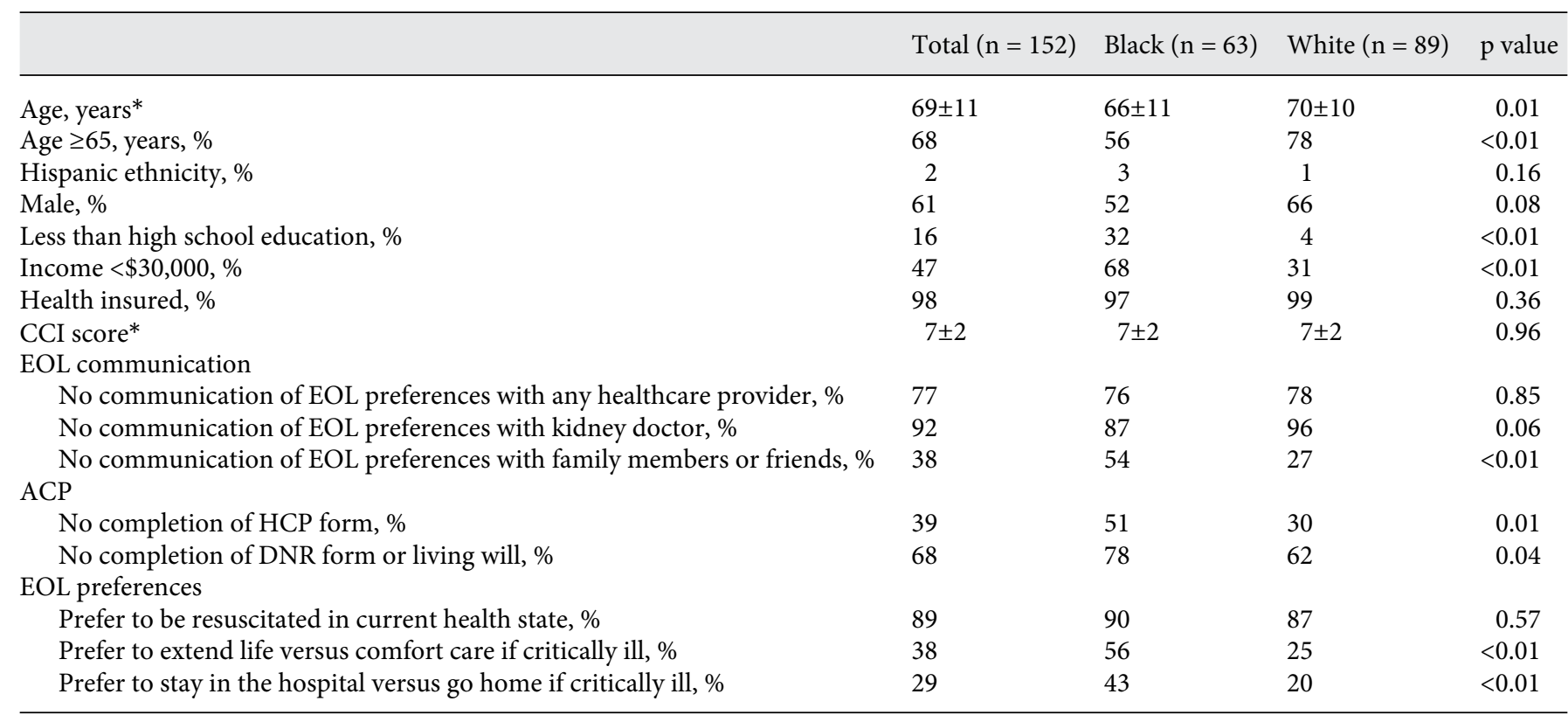

* Presented as mean \pm SD.

Table 2. Examples of hospice responses. ${ }^{1}$

\begin{tabular}{|c|c|c|}
\hline Poor knowledge & Partial knowledge & Good knowledge \\
\hline $\begin{array}{l}\text { It is a place where people who can not take } \\
\text { care of their activities of daily living are } \\
\text { taken care of }\end{array}$ & It means that you are seriously ill & $\begin{array}{l}\text { It is final care at home or in a suitable setting. It is } \\
\text { palliative care }\end{array}$ \\
\hline $\begin{array}{l}\text { The preface to hospital - a type of } \\
\text { hospital }\end{array}$ & $\begin{array}{l}\text { You usually have a } 6 \text { months window } \\
\text { of dying }\end{array}$ & Hospice is care that people get when they are dying \\
\hline
\end{tabular}

\footnotetext{
${ }^{1}$ Patient responses to 'since you have heard of hospice, what does this mean to you in your own words?'. Seventeen percent of Black patients had good knowledge vs. $61 \%$ of White patients $(\mathrm{p}<0.01)$.
}

analyses, Black patients were more likely to prefer to extend life if critically ill (OR 3.06, 95\% CI 1.23-7.60; table 3); however, there was no difference in preference for site of death.

In the final models, additional significant predictors included income ( $\mathrm{p}=0.03$; for EOL communication), study site ( $\mathrm{p}=0.02$; for completion of HCP form), Charlson Comorbidity Score $(\mathrm{p}<0.01$; for completion of HCP form) and education ( $\mathrm{p}=0.01$; for site of death). In multivariable analyses stratified by age, younger $(<65$ years) Black patients were more likely to prefer life-extending care (OR 9.15, 95\% CI 1.48-56.74; table 3) than White patients. However, there were no racial differences in preference for EOL communication, ACP or EOL care preferences among patients $\geq 65$ years of age (table 3). 
Table 3. EOL communication, ACP, and preferences for Black vs. White patients

\begin{tabular}{|c|c|c|c|c|}
\hline EOL preference & $\begin{array}{l}\text { Unadjusted OR } \\
(95 \% \mathrm{CI})\end{array}$ & $\begin{array}{l}\text { Adjusted } \\
\mathrm{OR}^{1}(95 \% \mathrm{CI})\end{array}$ & $\begin{array}{l}\text { Adjusted } \\
\mathrm{OR}^{2}(95 \% \mathrm{CI}) \\
<65 \text { years }\end{array}$ & $\begin{array}{l}\text { Adjusted } \\
\mathrm{OR}^{2}(95 \% \mathrm{CI}) \\
\geq 65 \text { years }\end{array}$ \\
\hline $\begin{array}{l}\text { No communication of EOL preferences with family } \\
\text { members of friends }\end{array}$ & $3.18(1.61-6.28)^{*}$ & $2.70(1.08-6.76)^{*}$ & $3.93(0.72-21.61)$ & $1.91(0.61-5.95)$ \\
\hline No completion of HCP form & $2.16(1.04-4.50)^{*}$ & $1.03(0.39-2.71)$ & $1.14(0.22-5.91)$ & $0.81(0.23-2.79)$ \\
\hline No completion of living will or $\mathrm{DNR}^{3}$ form & $2.37(1.21-4.63)^{*}$ & $2.13(0.77-5.90)$ & $4.05(0.27-59.63)$ & $1.68(0.53-5.33)$ \\
\hline Prefer to extend life vs. comfort care if critically ill & $3.66(1.85-7.26)^{*}$ & $3.06(1.23-7.60)^{*}$ & $9.15(1.48-56.74)^{*}$ & $2.40(0.74-7.76)$ \\
\hline Prefer to stay in the hospital vs. go home if critically ill & $2.66(1.31-5.40)^{*}$ & $1.87(0.71-4.75)$ & $6.49(0.78-54.15)$ & $1.45(0.45-4.74)$ \\
\hline
\end{tabular}

There were no significant racial differences with regards to the influence of religious/spiritual beliefs, cultural beliefs, and distrust of healthcare providers on preferences for care at the EOL (not displayed).

\section{Discussion}

Among patients with advanced CKD not yet on dialysis, few patients reported having discussions about EOL preferences and many had not completed any form of ACP. Black patients were less likely to understand the meaning of hospice compared to White patients. Furthermore, independent of age, education, income, comorbidities, study site and hospice knowledge, Black patients were less likely to communicate EOL preferences with family members and more likely to desire treatment intended to extend life if they were to become critically ill compared to White patients. In particular, younger Black patients were more likely to prefer life-extending care compared to younger White patients.

Our study confirms very low rates of ACP and EOL discussions for patients with CKD with their nephrologists and their other healthcare providers. Davison [7] showed that $44 \%$ of CKD patients had completed a healthcare proxy or enduring power of attorney and $38 \%$ reported having a personal directive; however, most of this cohort had already begun dialysis and was almost exclusively White. Furthermore, despite patients feeling somewhat comfortable discussing EOL issues with family members and healthcare providers, only $33 \%$ had spoken with family member/healthcare proxies and $10 \%$ had spoken with nephrologists within the past year about EOL issues. Our findings build upon this knowledge by enrolling a mixed race cohort of patients who had not yet begun dialysis, and by identifying the fact that Black patients in our study were less likely to communicate EOL preferences with their families and more likely to prefer life-extending treatment at the EOL compared to White patients. Factors such as lack of awareness of illness trajectory and prognosis in addition to unrealistic expectations of health statuses likely contribute to poor communication between patients with kidney disease and their families [28, 29]. For example, a recent randomized controlled trial of a communication intervention geared specifically toward Black patients with ESRD and their surrogates was particularly effective in improving patient-surrogate congruence and decisional confidence in goals of care [30]. This approach elucidated the complex relationships of health awareness and subsequent emotional burden that often occurs within families. As medical decision-making at the EOL for seriously ill patients can be difficult to predict and lead to traumatic experiences for patients' families and healthcare providers, the promotion of clear prognostic and ACP communication early in the course of CKD becomes a key strategy for improving delivery of EOL care while simultaneously reducing racial disparities [31-33].

Differences in EOL treatment preferences and practice patterns between different racial and ethnic groups have been confirmed across several disciplines of healthcare [34-37]. Such differences are inherently a problem if they are driven by misinformation. Fewer Black patients understood the meaning of hospice compared to White patients. Our data also demonstrated the important inter- 
play between hospice knowledge, racial differences, and EOL preferences - a notion that could contribute to subsequent discrepant hospice utilization at the EOL $[34,38]$. Through treatment of emotional and physical symptoms, hospice care has been proven to significantly improve the transition to death for patients and their families. Less understanding of the benefits of hospice has been associated with more aggressive care at EOL and a lower quality of death [31]. Educational programs for CKD patients and their families have the potential to promote more informed decision-making regarding treatment options at the EOL, better satisfaction with care, and decreased disparities [15].

We did not find substantial racial differences with regards to all aspects of care desired at the EOL. In adjusted analyses, Blacks and Whites had similar likelihood of completing HCP forms, DNR forms or living wills, and preference for site of death. Differences in nephrology clinic practices as well as illness severity could impact completion of advance directives. For instance, despite national guidelines that recommend the incorporation of ACP into routine clinical practice [13], none of the study sites had formal protocols for EOL communication or documentation. Factors such as education and income that remained significant predictors of certain EOL outcomes after adjustment may indeed represent social determinants of health that are fixed and unequal between the 2 races within the CKD population $[39,40]$. Historically, similar socioeconomic factors have been linked to racial differences in CKD development and progression. Our findings reinforce the need for more awareness in this arena with regards to disparate EOL care. Black patients were also younger than White patients in this cohort and our analyses revealed that racial differences in the outcomes examined were isolated to the younger patients. These findings could reflect ACP efforts traditionally targeted toward frail and older patients and highlight the need for interventions that reach a broader patient population $[2,14,41]$.

Our study has some limitations. The study population was comprised of Black and White English-speaking patients from 2 academic centers in the Boston metro area. This makes it difficult to generalize the findings across a wide range of population. We did not verify the documentation of EOL discussions in the EMR as all patients enrolled in the study were cognitively intact without dementia. While it is possible that patients may have misunderstood or not remembered previous EOL discussions, it is unlikely that this phenomenon explained the racial differences we observed. Also, previous work has cited strong religious/spiritual beliefs, culture, and healthcare distrust as catalysts for differences in preferences and between Black and White patients [21]. However, our findings showed no significant racial differences among these factors. We did not collect data on specific religion affiliation and therefore could not determine any overarching themes that would affect EOL preferences or communication relating to this factor. Also, due to the limited diversity of our cohort, we were unable to determine the effect of ethnicity on outcomes. Future research is needed to understand the divergence of our findings from prior work on racial disparities in religion/ spiritual beliefs, culture, and healthcare distrust. While this may reflect limitations in the generalizability of our cohort, these observations may also reflect true changes that have transpired over time and/or the possibility that racial disparities in these factors may not be present among CKD patients or in general for patients who have had stable access to medical care (as has been the case in Massachusetts). Additionally, we explored the concept of hospice as a follow-up question only with patients who reported that they were familiar with the term. This could have potentially excluded patients who may have been knowledgeable about the concept despite unfamiliarity with the word hospice. Furthermore, sociocultural factors such as education level could have affected patients' likelihood of answering a question phrased as 'Have you heard of...?' with a 'yes' or 'no' response. Independent of actual understanding of the meaning of 'hospice', it might be harder for people with a higher level of education to say that they have never heard of this word. Another limitation relates to the cross-sectional study design, which blocks our ability to make stronger causal inferences.

Despite these limitations, as far as our knowledge goes, this study is the first to investigate and describe racial differences in EOL communication and preferences among pre-dialysis patients with CKD. In conclusion, EOL discussions with providers and the rate of ACP are very low for patients with CKD. Black patients are less likely to have had any EOL communication with their families and prefer more aggressive care at the EOL than White patients. At the same time, Black patients in our study have lower hospice knowledge compared to White patients. This may have a large influence on the racial disparities in EOL preferences we observe. These differences have important implications in designing research and clinical care interventions that improve patient understanding, promote better informed decision-making and reduce racial disparities for patients with advanced CKD. 


\section{Acknowledgments}

We thank Sarah M. Doughtery for her assistance with patient recruitment and data entry. This study was supported by a grant from the American Society of Nephrology Foundation for Kidney Research Ben J. Lipps Research Fellowship Program (PI - Eneanya) and a mentorship grant from the National Institute of Diabetes and Digestive and Kidney Diseases K24-DK094872 (PI - Thadhani).

\section{Disclosure Statement}

All authors have approved this manuscript for submission. To the best of our knowledge, no conflict of interest, financial or otherwise, exists.

\section{References}

1 Saran R, Li Y, Robinson B, Ayanian J, Balkrishnan R, Bragg-Gresham J, Chen JT, Cope E, Gipson D, He K, Herman W, Heung M, Hirth RA, Jacobsen SS, Kalantar-Zadeh K, Kovesdy CP, Leichtman AB, Lu Y, Molnar MZ, Morgenstern H, Nallamothu B, O’Hare AM, Pisoni R, Plattner B, Port FK, Rao P, Rhee CM, Schaubel DE, Selewski DT, Shahinian V, Sim JJ, Song P, Streja E, Kurella Tamura M, Tentori F, Eggers PW, Agodoa LY, Abbott KC: US renal data system 2014 annual data report: epidemiology of kidney disease in the United States. Am J Kidney Dis 2015; 65(1 suppl 1):Svii, S1-S305.

2 Wong SP, Kreuter W, O'Hare AM: Treatment intensity at the end of life in older adults receiving long-term dialysis. Arch Intern Med 2012;172:661-663; discussion 663-664.

3 Moss AH, Holley JL, Upton MB: Outcomes of cardiopulmonary resuscitation in dialysis patients. J Am Soc Nephrol 1992;3:12381243.

4 Wong SP, Kreuter W, Curtis JR, Hall YN, O'Hare AM: Trends in in-hospital cardiopulmonary resuscitation and survival in adults receiving maintenance dialysis. JAMA Intern Med 2015;175:1028-1035.

5 Saeed F, Adil MM, Malik AA, Schold JD, Holley JL: Outcomes of in-hospital cardiopulmonary resuscitation in maintenance dialysis patients. J Am Soc Nephrol 2015;26:3093-3101.

6 Goff SL, Eneanya ND, Feinberg R, Germain MJ, Marr L, Berzoff J, Cohen LM, Unruh M: Advance care planning: a qualitative study of dialysis patients and families. Clin J Am Soc Nephrol 2015;10:390-400.

7 Davison SN: End-of-life care preferences and needs: perceptions of patients with chronic kidney disease. Clin J Am Soc Nephrol 2010; 5:195-204.

8 Detering KM, Hancock AD, Reade MC, Silvester W: The impact of advance care planning on end of life care in elderly patients: randomised controlled trial. BMJ 2010; 340:c1345.

9 Schonwetter RS, Walker RM, Solomon M, Indurkhya A, Robinson BE: Life values, resuscitation preferences, and the applicability of living wills in an older population. J Am Geriatr Soc 1996;44:954-958.

10 Song MK, Ward SE, Fine JP, Hanson LC, Lin FC, Hladik GA, Hamilton JB, Bridgman
JC: Advance care planning and end-of-life decision making in dialysis: a randomized controlled trial targeting patients and their surrogates. Am J Kidney Dis 2015;66:813822 .

11 Bristowe K, Horsley HL, Shepherd K, Brown H, Carey I, Matthews B, O’Donoghue D, Vinen K, Murtagh FE: Thinking ahead - the need for early advance care planning for people on haemodialysis: a qualitative interview study. Palliat Med 2015;29:443-450.

12 Tong A, Cheung KL, Nair SS, Kurella Tamura M, Craig JC, Winkelmayer WC: Thematic synthesis of qualitative studies on patient and caregiver perspectives on end-of-life care in CKD. Am J Kidney Dis 2014;63:913-927.

13 Davison SN, Levin A, Moss AH, Jha V, Brown EA, Brennan F, Murtagh FE, Naicker S, Germain MJ, O’Donoghue DJ, Morton RL, Obrador GT; Kidney Disease: Improving Global Outcomes: Executive summary of the KDIGO controversies conference on supportive care in chronic kidney disease: developing a roadmap to improving quality care. Kidney Int 2015;88:447-459.

14 Kurella Tamura M, Goldstein MK, Pérez-Stable EJ: Preferences for dialysis withdrawal and engagement in advance care planning within a diverse sample of dialysis patients. Nephrol Dial Transplant 2010;25:237-242.

15 Davison SN, Jhangri GS, Koffman J: Knowledge of and attitudes towards palliative care and hospice services among patients with advanced chronic kidney disease. BMJ Support Palliat Care 2016;6:66-74.

16 Fried TR, Redding CA, Robbins ML, Paiva AL, O'Leary JR, Iannone L: Development of personalized health messages to promote engagement in advance care planning. J Am Geriatr Soc 2016;64:359-364.

17 Thomas BA, Rodriguez RA, Boyko EJ, Robinson-Cohen C, Fitzpatrick AL, O'Hare AM: Geographic variation in black-white differences in end-of-life care for patients with ESRD. Clin J Am Soc Nephrol 2013;8:11711178.

18 Barnato AE, Chang CC, Saynina O, Garber AM: Influence of race on inpatient treatment intensity at the end of life. J Gen Intern Med 2007;22:338-345.

19 Jennings B, Ryndes T, D’Onofrio C, Baily MA: Access to hospice care. Expanding boundaries, overcoming barriers. Hastings Cent Rep 2003; (suppl):S3-S7, S9-S13, S15S21 passim.

20 NHPCO Facts and Figures: Hospice Care in America. Alexandria, National Hospice and Palliative Care Organization, 2014.

21 Crawley L, Payne R, Bolden J, Payne T, Washington $\mathrm{P}$, Williams $\mathrm{S}$; Initiative to Improve Palliative and End-of-Life Care in the African American Community: Palliative and end-oflife care in the African American community. JAMA 2000;284:2518-2521.

22 Volandes AE, Paasche-Orlow M, Gillick MR Cook EF, Shaykevich S, Abbo ED, Lehmann L: Health literacy not race predicts end-of-life care preferences. J Palliat Med 2008;11:754762.

23 Levey AS, Bosch JP, Lewis JB, Greene T, Rogers N, Roth D: A more accurate method to estimate glomerular filtration rate from serum creatinine: a new prediction equation. Modification of diet in renal disease study group. Ann Intern Med 1999;130:461-470.

24 Pfeiffer E: A short portable mental status questionnaire for the assessment of organic brain deficit in elderly patients. J Am Geriatr Soc 1975;23:433-441.

25 Charlson ME, Pompei P, Ales KL, MacKenzie CR: A new method of classifying prognostic comorbidity in longitudinal studies: development and validation. J Chronic Dis 1987;40: 373-383.

26 A controlled trial to improve care for seriously ill hospitalized patients. The study to understand prognoses and preferences for outcomes and risks of treatments (SUPPORT). The SUPPORT principal investigators. JAMA 1995;274:1591-1598.

27 Shelkowitz E, Vessella SL, O'Reilly P, Tucker $\mathrm{R}$, Lechner BE: Counseling for personal care options at neonatal end of life: a quantitative and qualitative parent survey. BMC Palliat Care 2015; 14:70.

28 Wachterman MW, Marcantonio ER, Davis RB, Cohen RA, Waikar SS, Phillips RS, McCarthy EP: Relationship between the prognostic expectations of seriously ill patients undergoing hemodialysis and their nephrologists. JAMA Intern Med 2013;173:1206-1214.

29 Paterson BL: The shifting perspectives model of chronic illness. J Nurs Scholarsh 2001;33: 21-26. 
30 Song MK, Ward SE, Happ MB, Piraino B, Donovan HS, Shields AM, Connolly MC: Randomized controlled trial of SPIRIT: an effective approach to preparing African-American dialysis patients and families for end of life. Res Nurs Health 2009;32:260-273.

31 Teno JM, Clarridge BR, Casey V, Welch LC, Wetle T, Shield R, Mor V: Family perspectives on end-of-life care at the last place of care. JAMA 2004;291:88-93.

32 Uhlmann RF, Pearlman RA, Cain KC: Physicians' and spouses' predictions of elderly patients' resuscitation preferences. J Gerontol 1988;43:M115-M121.

33 Mack JW, Paulk ME, Viswanath K, Prigerson HG: Racial disparities in the outcomes of communication on medical care received near death. Arch Intern Med 2010;170:1533-1540.
34 Zheng NT, Mukamel DB, Caprio T, Cai S, Temkin-Greener H: Racial disparities in inhospital death and hospice use among nursing home residents at the end of life. Med Care 2011;49:992-998.

35 Loggers ET, Maciejewski PK, Jimenez R, Nilsson M, Paulk E, Stieglitz H, Prigerson HG: Predictors of intensive end-of-life and hospice care in Latino and white advanced cancer patients. J Palliat Med 2013;16:12491254.

36 Hernandez RA, Hevelone ND, Lopez L, Finlayson SR, Chittenden E, Cooper Z: Racial variation in the use of life-sustaining treatments among patients who die after major elective surgery. Am J Surg 2015;210:52-58.

37 Dillon PJ, Roscoe LA: African Americans and hospice care: a narrative analysis. Narrat Inq Bioeth 2015;5:151-165.
38 Cagle JG, LaMantia MA, Williams SW, Pek J, Edwards LJ: Predictors of preference for hospice care among diverse older adults. Am J Hosp Palliat Care 2016;33:574-584.

39 Nicholas SB, Kalantar-Zadeh K, Norris KC: Socioeconomic disparities in chronic kidney disease. Adv Chronic Kidney Dis 2015;22:615.

40 Bruce MA, Beech BM, Sims M, Brown TN, Wyatt SB, Taylor HA, Williams DR, Crook E: Social environmental stressors, psychological factors, and kidney disease. J Investig Med 2009;57:583-589.

41 Kurella M, Covinsky KE, Collins AJ, Chertow GM: Octogenarians and nonagenarians starting dialysis in the United States. Ann Intern Med 2007;146:177-183. 